\title{
Phân tích hiệu quả kỹ thuật của các ngân hàng thương mại cổ phần tại Việt Nam
}

\section{Analysing technical efficiency of joint stock commercial banks in Vietnam}

\author{
Nguyễn Minh Kiều ${ }^{1 *}$, Nguyễn Ngọc Thùy Trang ${ }^{2}$ \\ ${ }^{1}$ Trường Đại học Mở Thành phố Hồ Chí Minh, Việt Nam \\ ${ }^{2} \mathrm{Ngân}$ hàng Eximbank, Việt Nam \\ *Tác giả liên hệ, Email: kieu.nm@ou.edu.vn
}

\section{THÔNG TIN}

DOI: $10.46223 / \mathrm{HCMCOUJS}$. econ.vi.15.3.1330.2020

Ngày nhận: 19/11/2019

Ngày nhận lại: 13/05/2020

Duyệt đăng: 14/05/2020

Tù khóa:

hiệu quả kỹ thuật, hiệu quả hoạt động, phân tích biên ngẫu nhiên (SFA), phân tích bao dữ liệu (DEA)

\section{Keywords:}

Technical Efficiency (TE), bank efficiency, Stochastic Frontier Analysis (SFA), Data Envelopment Analysis (DEA)

\section{TÓM TĂT}

Hiệu quả hoạt động luôn được xem là một yếu tố quyết định năng lực cạnh tranh của bất kỳ ngân hàng nào trên thị trường. Do vậy, nghiên cứu này tiến hành đánh giá mức hiệu quả của các NHTMCP Việt Nam đạt được trong giai đoạn 2011 - 2018. Trong đó, hiệu quả hoạt động được ước lượng thông qua phương pháp phân tích hiệu quả kỹ thuật bằng phân tích biên ngẫu nhiên (SFA) và phân tích bao dữ liệu (DEA). Đồng thời, nghiên cứu cũng sử dụng phương pháp bình phương tối thiểu gộp (Pooled OLS), mô hình tác động ngẫu nhiên (FEM), phương pháp ước lượng bình phương nhỏ nhất (GLS) để đánh giá tác động của các nhân tố đến hiệu quả kỹ thuật của các NHTMCP Việt Nam. Kết quả chỉ ra rằng, các yếu tố thuộc nhóm hiệu quả quản lý (ROA, ETA) có ảnh hưởng tích cực đến hiệu quả kỹ thuật hay hiệu quả hoạt động của các NHTMCP Việt Nam.

ABSTRACT
Bank efficiency is always the determiants of the
competitiveness of any banks in the market. Therefore, this
article is to study the effectiveness of Joint Stock Commercial
Banks in Vietnam achieved in 2011 - 2018. Specially, banking
performance is estimated by technical efficiency: Stochastic
Frontier Analysis (SFA) and Data Envelopment Analysis
(DEA). Furthermore, the study also uses Pooled OLS method,
Fixed Effect model (FEM), Generalized Least Squares method
(GLS) to assess the impact of factors to technical efficiency of
Joint Stock Commercial Banks. The study results showed that
the factors of effective management (ROA, ETA) have
significantly positive impact on technical efficiency or
performance of Joint Stock Commercial Banks.

\section{Giới thiệu}

Với nhiệm vụ là trung gian luân chuyển vốn của nền kinh tế, các NHTMCP luôn có vai trò quan trọng trong hệ thống tài chính của bất kỳ quốc gia nào, đặc biệt là tại các nước đang phát 
triển như Việt Nam. Theo báo cáo của Ngân hàng Nhà nước Việt Nam (2018), số lượng các NHTMCP tại Việt Nam năm 2014 là 34 ngân hàng thì tính đến thời điểm 30/06/2018, số lượng NHTMCP đang hoạt động chỉ còn 31 ngân hàng. Con số này là một dẫn chứng cho thấy những ngân hàng nào hoạt động không hiệu quả, không có khả năng cạnh tranh đã dần được thay thế bằng những ngân hàng khác hoạt động tốt hơn. Do vậy, có thể nói hiệu quả là một trong những tiêu chí quan trọng, buộc các NHTMCP phải đánh giá để tồn tại và phát triển, đặc biệt là trong môi trường cạnh tranh quốc tế ngày một gia tăng như hiện nay.

Hiện tại, đã có tương đối nhiều nghiên cứu quan tâm đến vấn đề hiệu quả hoạt động ngân hàng theo cả hai hướng định tính và định lượng. Với hướng nghiên cứu định lượng, xu hướng phương pháp đánh giá hiệu quả hoạt động mới đang được sử dụng phổ biến trong những năm gần đây đó chính là phương pháp phân tích hiệu quả kỹ thuật thay vì phân tích chỉ số tài chính thông thường. Tuy nhiên, hầu hết các bài phân tích chỉ sử dụng một trong hai phương pháp DEA hoặc SFA, ít có bài viết nào sử dụng đồng thời cả hai phương pháp DEA và SFA để đánh giá hiệu quả kỹ thuật của các NHTMCP Việt Nam đạt được, đặc biệt là trong giai đoạn gần đây. Chính vì vậy, việc nghiên cứu hiệu quả hoạt động của các NHTMCP hay cụ thể hơn là xác định mức hiệu quả kỹ thuật mà các NHTMCP đạt được là hết sức cần thiết.

Mục tiêu của nghiên cứu này là xác định hiệu quả hoạt động của các NHTMCP tại Việt Nam, trong đó hiệu quả hoạt động được ước lượng thông qua phương pháp phân tích hiệu quả kỹ thuật bằng hai mô hình chính SFA và $\mathrm{DEA}$, đồng thời kiểm định các nhân tố tác động đến hiệu quả kỹ thuật của NHTMCP. Từ đó có thể bổ sung bằng chứng nghiên cứu thực nghiệm, kênh thông tin tham khảo về đánh giá hiệu quả hoạt động, giúp các nhà phân tích, quản trị ngân hàng có thể đưa ra chính sách phù hợp, góp phần nâng cao vị thế cạnh tranh của ngân hàng mình trên thị trường tài chính.

\section{Cơ sở lý thuyết và các nghiên cứu có liên quan}

\subsection{Co' sở lý thuyết}

Coelli, O’ Donnell, và Battese (2005) đã đưa ra định nghĩa về hiệu quả như sau: Hiệu quả là năng lực hoạt động của ngân hàng khi đạt được mức sản lượng tối đa với đơn vị đầu vào cho trước. Như vậy, với hoạt động của các NHTMCP hiệu quả được hiểu là mối liên hệ giữa các yếu tố đầu ra (outputs) và các yếu tố đầu vào (inputs) mà ngân hàng đã bỏ ra để đạt mức khả năng sinh lời mong muốn. Ta có thể tạm chia hiệu quả thành hai nhóm phổ biến như sau: Nhóm hiệu quả tài chính và nhóm hiệu quả kỹ thuật.

Bản chất của hiệu quả tài chính chính là hiệu quả kinh doanh của một ngân hàng. Hiệu quả kinh doanh là chỉ tiêu kinh tế tổng hợp phản ánh trình độ sử dụng các yếu tố của quá trình sản xuất bao gồm các nguồn vật lực, nguồn tài chính để đạt được mức hiệu quả yêu cầu (P. N. Nguyen, 2013). Một số chỉ tiêu phổ biến thường được sử dụng để phản ánh hiệu quả kinh doanh như: ROE, ROS, ROI, NIM,... Tuy nhiên, các chỉ số tài chính này lại thường gặp một số hạn chế khi sử dụng như: Chỉ phản ánh mối liên hệ tương quan giữa hai biến số nên không thể đưa ra nhận định tổng quát về tình trạng của đối tượng đang được đánh giá, buộc nhà phân tích khi sử dụng cần phải kết hợp so sánh nhiều chỉ số, dẫn đến việc lựa chọn chỉ số tính toán sử dụng còn mang tính chủ quan; phải sử dụng chính xác tử số hoặc mẫu số trong công thức tính toán, chuẩn mực kế toán hay phương pháp kế toán cần có sự thống nhất giữa các đối tượng so sánh; các giá trị dị biệt nếu có xuất hiện trong dữ liệu chỉ số tài chính sẽ mắc phải lỗi phương sai sai số thay đổi (Faello, 2015). Do đó, việc tìm kiếm sử dụng phương pháp mới, mang tính tổng quát, không những đánh giá được hiệu quả hoạt động mà còn cho biết bức tranh tổng quan về thứ hạng của ngân hàng đánh giá đó chính là phương pháp phân tích hiệu quả kỹ thuật. 
Cùng với khái niệm về đường giới hạn khả năng sản xuất $(\mathrm{PPF})$, khái niệm về hiệu quả kỹ thuật đã xuất hiện một thời gian dài trong kinh tế học cho đến nay. Theo Koopmans (1957), hiệu quả kỹ thuật lần đầu tiên đã được định nghĩa như sau: Khi và chỉ khi điểm hiệu quả ngân hàng đạt được khả thi và không tồn tại điểm nào khác "tốt hơn" điểm đó thì ngân hàng đạt được hiệu quả kỹ thuật. Farrell (1957) đã đưa ra một định nghĩa khác dưới cách tiếp cận đầu ra (hay còn gọi là tối đa hóa đầu ra) và được chấp nhận rộng rãi. Hiệu quả kỹ thuật bao gồm hiệu quả kỹ thuật thuần túy (PTE) và hiệu quả quy mô (SE). Coelli và cộng sự (2005) đã bổ sung góp phần hoàn thiện định nghĩa hiệu quả kỹ thuật theo cách tiếp cận tối đa hóa đầu vào đó là với lượng đầu vào tối thiểu, một ngân hàng có thể sản xuất được một lượng đầu ra cố định thì ngân hàng đó đạt hiệu quả kỹ thuật.

Được áp dụng từ năm 1970, khung phân tích CAMELS (hoặc CAMEL) là hệ thống xếp hạng, giám sát tình hình ngân hàng của Mỹ được nhiều tổ chức tín dụng trên thế giới sử dụng như là chuẩn mực dùng để đánh giá hiệu quả, quản trị rủi ro của các ngân hàng nói riêng và các tổ chức tín dụng nói chung. Khung phân tích bao gồm sáu yếu tố cơ bản: $\mathrm{C}$ - Mức an toàn vốn, $\mathrm{A}$ - Chất lượng tài sản, $M$ - Khả năng quản lý, $\mathrm{E}$ - Thu nhập, $\mathrm{L}$ - Tính thanh khoản và $\mathrm{S}$ - Độ nhạy cảm với rủi ro thị trường. Tiêu chuẩn này đã được nhiều nhà nghiên cứu sử dụng để lựa chọn các biến phản ánh đến hiệu quả của ngân hàng như H. V. Nguyen (2008), Chen, Hu, và Su (2006). Tuy nhiên, việc lựa chọn biến nào phù hợp còn dựa trên mục tiêu của nhà phân tích, lãnh đạo ngân hàng và người nghiên cứu. Do đó, dựa trên khung phân tích CAMELS và từ những nghiên cứu trước, bài viết này tiến hành xem xét mối liên hệ giữa năm nhân tố quan trọng, bao gồm Quy mô ngân hàng, ROA, NPL, DLR, ETA lên hiệu quả của các NHTMCP tại Việt Nam.

\subsection{Các nghiên cúu liên quan}

Bất kỳ một doanh nghiệp nào hoạt động trên thị trường luôn có mục tiêu sau cùng đó là tối đa hóa giá trị tài sản cho chủ sở hữu, cổ đông và ngân hàng cũng không ngoại lệ. Vì vậy, việc xem xét, đánh giá hiệu quả hoạt động luôn là vấn đề cấp thiết, được đặt lên hàng đầu của mỗi đơn vị và luôn được nhiều nhà nghiên cứu quan tâm, một số nghiên cứu trong và ngoài nước đã đề cập đến vấn đề hiệu quả hoạt động ngân hàng, đặc biệt là phương pháp phân tích hiệu quả kỹ thuật trong những năm gần đây.

Pham và Chau (2011) chỉ ra rằng các NHTM Nhà nước mặc dù đạt mức dư nợ tín dụng cao hơn khối NHTMCP nhưng mức hiệu quả kỹ thuật về thu nhập đạt được lại tương đương với khối NHTMCP. Michael (2016) nghiên cứu 20 ngân hàng nông thôn và ngân hàng cộng đồng tại Ghana có mức hiệu quả kỹ thuật tương đối tốt. Phương pháp DEA và mô hình hồi quy được sử dụng cho thấy các yếu tố như: Quy mô, lợi nhuận, chất lượng nguồn vốn ảnh hưởng mạnh mẽ đến hiệu quả kỹ thuật của các ngân hàng này. Ngoài ra, hai yếu tố quy mô và chất lượng nguồn vốn dẫn đến giảm hiệu quả kỹ thuật, trong khi sự gia tăng lợi nhuận sẽ giúp cho ngân hàng nông thôn đạt hiệu quả kỹ thuật cao hơn.

K. Q. Nguyen (2016) đã chứng minh sự hiệu quả khác biệt giữa top 10 ngân hàng hiệu quả cao và top 10 ngân hàng hiệu quả thấp (các ngân hàng hiệu quả cao được phân thành các tầng cao hơn và các ngân hàng hiệu quả thấp được phân thành các tầng thấp hơn) thông qua dữ liệu nghiên cứu được thu thập từ 20 NHTM Việt Nam năm 2014. T. T. T. Nguyen (2017) đã chứng minh các NHTM sử dụng tương đối hiệu quả các nguồn lực đầu vào với phương pháp phân tích bao dữ liệu DEA và mô hình hồi quy Tobit được sử dụng để đánh giá hiệu quả hoạt động của 21 NHTM trên địa bàn tỉnh Thái Nguyên giai đoạn 2011 - 2015 và phân tích tác động của các nhân tố riêng, mang tính đặc trưng của ngân hàng, các điều kiện thị trường khác đến hoạt động hiệu quả của các NHTM.

Nhìn chung, phương pháp phân tích hiệu quả kỹ thuật để đánh giá hiệu quả hoạt động của 
đối tượng ngân hàng đang được nhiều nhà phân tích quan tâm sử dụng. Tuy nhiên, các nghiên cứu trước chủ yếu sử dụng một trong hai phương pháp đặc trưng là DEA hoặc SFA. Một số nghiên cứu có đi sâu hơn tiến hành kết hợp phân tích hồi quy để đánh giá thêm mức độ tác động của các nhân tố đến hiệu quả ngân hàng và dữ liệu điều tra cũng đã cũ. Do vậy, bài viết này tiến hành nghiên cứu hiệu quả hoạt động đối tượng các NHTMCP tại Việt Nam bằng phương pháp phân tích hiệu quả kỹ thuật theo mô hình tham số và phi tham số là SFA và DEA kết hợp, đồng thời phân tích hồi quy dữ liệu bảng được thực hiện tiếp tục ở bước hai nhằm xác định và đánh giá mức độ tác động của các nhân tố đến hiệu quả kỹ thuật của loại hình ngân hàng này để có thể đưa ra được bức tranh tổng quát về tình hình hoạt động của các NHTMCP tại Việt Nam trong những năm gần đây.

\section{Mô hình và phương pháp nghiên cứu}

\subsection{Mô hình nghiên cúu}

Từ các lý thuyết và nghiên cứu trước (Coelli et al., 2005; Eva, 2018; Farrell, 1957; Gamachis, 2016), nghiên cứu đề xuất mô hình phân tích hiệu quả kỹ thuật và kiểm định các nhân tố tác động đến hiệu quả kỹ thuật các NHTMCP tại Việt Nam thông qua các bước như sau:

- Bước 1: Đánh giá hiệu quả kỹ thuật NHTMCP Việt Nam

Hiệu quả hoạt động ngân hàng được xác định thông qua phương pháp phân tích kỹ thuật bằng hai mô hình chính: Mô hình phân tích biên ngẫu nhiên SFA và mô hình phân tích bao dữ liệu DEA với giả thuyết định hướng đầu vào. Các yếu tố đầu ra, đầu vào được xác định dựa trên quan điểm của Berger và Humphrey (1997) xem ngân hàng là một tổ chức trung gian tài chính. Bảng 1 trình bày tổng hợp các yếu tố đầu ra, đầu vào được lựa chọn trong nghiên cứu:

\section{Bảng 1}

Bảng tổng hợp các yếu tố đầu vào - đầu ra

\begin{tabular}{|c|c|c|c|c|}
\hline \multicolumn{2}{|c|}{ Các yếu tố } & & $\begin{array}{c}\text { Đơn vị } \\
\text { tính }\end{array}$ & Nghiên cứu trước \\
\hline \multicolumn{5}{|l|}{ Đầu vào } \\
\hline \multicolumn{2}{|c|}{ Tài sản cố định ròng } & $\begin{array}{l}\mathrm{K}=\text { Nguyên giá tài } \\
\text { sản cố đị̣nh - Hao } \\
\text { mòn lũy kế }\end{array}$ & $\begin{array}{l}\text { Triệu } \\
\text { đồng }\end{array}$ & $\begin{array}{l}\text { T. T. H. Nguyen và Le (2018), Eva } \\
\text { (2018), K. Q. Nguyen (2016), Huynh } \\
\text { (2015), Inès (2013), H. V. Nguyen (2008) }\end{array}$ \\
\hline \multicolumn{2}{|l|}{ Lao động } & $\begin{array}{l}\mathrm{L}=\text { Tổng chi phí } \\
\text { lương }\end{array}$ & $\begin{array}{l}\text { Triệu } \\
\text { đồng }\end{array}$ & $\begin{array}{l}\text { T. T. H. Nguyen và Le (2018), Eva } \\
\text { (2018), Gamachis (2016), Huynh (2015) }\end{array}$ \\
\hline \multicolumn{2}{|c|}{ Tiền gửi huy động } & $\begin{array}{l}\mathrm{D}=\text { Tiền gửi khách } \\
\text { hàng }+ \text { Tiền gửi của } \\
\text { tổ chức tín dụng khác }\end{array}$ & $\begin{array}{l}\text { Triệu } \\
\text { đồng }\end{array}$ & $\begin{array}{l}\text { T. T. H. Nguyen và Le (2018), T. T. T. } \\
\text { Nguyen (2017), K. Q. Nguyen (2016), } \\
\text { Eva (2018), Gamachis (2016), Huynh } \\
(2015)\end{array}$ \\
\hline \multicolumn{5}{|l|}{ Đầu ra } \\
\hline \multirow{2}{*}{$\begin{array}{l}\text { Thu nhập } \\
\text { hoạt động } \\
\text { (Y) }\end{array}$} & $\begin{array}{l}\text { Thu nhập } \\
\text { lãi }\end{array}$ & $\mathrm{Y}_{1}$ & $\begin{array}{l}\text { Triệu } \\
\text { đồng }\end{array}$ & $\begin{array}{l}\text { T. T. H. Nguyen và Le (2018), Phan và } \\
\text { Tran (2017), Huynh (2015) }\end{array}$ \\
\hline & $\begin{array}{l}\text { Thu nhập } \\
\text { ngoài lãi }\end{array}$ & $\mathrm{Y}_{2}$ & $\begin{array}{l}\text { Triệu } \\
\text { đồng }\end{array}$ & $\begin{array}{l}\text { T. T. H. Nguyen và Le (2018), ), Phan và } \\
\text { Tran (2017), Huynh (2015) }\end{array}$ \\
\hline
\end{tabular}

Nguồn: Kết quả phân tích dữ liệu của nhóm nghiên cứu 


\subsubsection{Mô hình ước lượng hiệu quả kỹ thuật bằng phương pháp phân tích biên ngẫu nhiên (SFA)}

Với phương pháp SFA, việc xác định hàm sản xuất cụ thể và mang tính đặc trưng, tổng quát cho hoạt động của đối tượng nghiên cứu là vấn đề quan trọng nhất. Do đó, nghiên cứu sẽ tiến hành thực hiện kiểm định thống kê tỷ số hợp lý LR-test (generalised log-likehood ratio test) để kiểm định dạng hàm phù hợp. Hai dạng hàm được xem xét là hàm sản xuất Cobb-Douglas và hàm Translog. Ngoài ra, tỷ số này còn được dùng để kiểm định có hay không phi hiệu quả kỹ thuật và phi hiệu quả kỹ thuật thay đổi bất biến theo thời gian.

(1) Kiểm định dạng hàm sản xuất:

- Ước lượng hàm ước lượng hàm sản xuất Cobb-Douglas dạng:

$$
\ln Y_{\text {it }}=\alpha_{0}+\alpha_{1} \ln K_{\text {it }}+\alpha_{2} \ln L_{\text {it }}+\alpha_{3} \ln D_{\text {it }}+v_{\text {it }}-u_{\text {it }}
$$

- Ước lượng hàm ước lượng hàm sản xuất Translog dạng:

$$
\begin{aligned}
& \ln Y_{i t}=\alpha_{0}+\alpha_{1} \ln K_{i t}+\alpha_{2} \ln L_{i t}+\alpha_{3} \ln D_{i t}++\beta_{1} \ln K_{i t} L_{i t}+\beta_{2} \ln K_{i t} D_{i t} \\
& +\beta_{3} \ln L_{i t} D_{i t}+\lambda_{1} \ln K^{2}{ }_{i t}+\lambda_{2} \operatorname{lnL}^{2}{ }_{i t}+\lambda_{3} \operatorname{lnD}^{2}{ }_{i t}+v_{i t}-u_{i t}
\end{aligned}
$$

Trong đó:

$\ln Y_{i t}$ : Logarit tự nhiên của giá trị gia tăng hoặc doanh thu thuần của ngân hàng i năm $\mathrm{t}$.

lnK $K_{i t}$ : Logarit tự nhiên vốn ròng của ngân hàng i năm $\mathrm{t}$.

$\operatorname{lnL} L_{i t}$ : Logarit tự nhiên lao động của ngân hàng i năm t.

Giả thuyết $\mathrm{H}_{\mathrm{o}}$ được đưa ra như sau: Hàm sản xuất Cobb-Douglas phù hợp với bộ dữ liệu hay $\mathrm{H}_{0:} \beta_{1}=\beta_{2}=\beta_{3}=\lambda_{1}=\lambda_{2}=\lambda_{3}=0$. Thống kê kiểm định LR-test sẽ tuân theo phân phối Chi bình phương hỗn hợp với bậc tự do $\mathrm{df}=6$. Nếu giá trị LR-test lớn hơn giá trị tới hạn với mức ý nghĩa lần lượt là $1 \%$ và $5 \%$ thì giả thuyết $\mathrm{H}_{0}$ bị bác bỏ, dạng hàm Translog được chọn là phù hợp và ngược lại.

\section{(2) Kiểm định có hay không phi hiệu quả kỹ thuật:}

Giả thuyết $\mathrm{H}_{0}$ : Không có phi hiệu quả kỹ thuật. Nếu chấp nhận $\mathrm{H}_{0}$, hàm hợp lý ứng với ước lượng OLS và chênh lệch giữa sản lượng tối đa và sản lượng quan sát được là do ảnh hưởng của phần dư (nhiễu). LR-test tuân theo phân phối Chi bình phương hỗn hợp với bậc tự do $\mathrm{df}=3$.

(3) Kiểm định phi hiệu quả kỹ thuật thay đổi theo thời gian:

Giả thuyết $\mathrm{H}_{0}$ : Phi hiệu quả kỹ thuật không thay đổi theo thời gian. Với bậc tự do $\mathrm{df}=1$, nếu giá trị LR-test lớn hơn giá trị tới hạn với mức ý nghĩa $1 \%$ và $5 \%$ thì giả thuyết $\mathrm{H}_{0}$ bị bác bỏ, nghĩa là phi hiệu quả kỹ thuật thay đổi theo thời gian và ngược lại, ta chấp nhận nhiễu phi hiệu quả kỹ thuật không thay đổi theo thời gian.

Dựa trên quan điểm của Aigner và Schmidt (1977), mô hình hàm sản xuất biên ngẫu nhiên bao gồm phần sai số của mô hình được phân tách thành hai phần: (1) Đại diện cho phân phối ngẫu nhiên đối xứng không quan sát được (v) và phần còn lại do (2) phi hiệu quả kỹ thuật gây ra (u) có dạng như sau:

$$
\ln (\mathrm{y})=\mathrm{x}_{\mathrm{i}} \beta+\mathrm{v}_{\mathrm{i}}-\mathrm{u}_{\mathrm{i}}
$$

Hiệu quả kỹ thuật của mỗi ngân hàng được xác định:

$$
\mathrm{TE}_{\mathrm{i}}=\mathrm{E}\left(\exp \left\{-\mathrm{u}_{\mathrm{i}}\right\} \varepsilon_{\mathrm{i}}\right)=\left[\frac{1-\Phi\left(\sigma_{*}-\frac{\mu_{* i}}{\sigma_{*}}\right)}{1-\Phi\left(-\frac{\mu_{* i}}{\sigma_{*}}\right)}\right] \cdot \exp \left\{-\mu_{* i}+\frac{1}{2} \sigma_{*}^{2}\right\}
$$




\subsubsection{Mô hình ước lượng hiệu quả kỹ thuật bằng phưong pháp bao dũ liệu (DEA)}

Với đối tượng nghiên cứu là các NHTMCP, đơn vị kinh doanh chứa một lượng lớn giao dịch lẫn các yếu tố đầu vào và đầu ra hàng ngày thì giả định hiệu quả không đổi theo quy mô (CRS) là không thể. Do đó, nghiên cứu sẽ thực hiện theo giả định mô hình định hướng đầu vào hiệu suất thay đổi theo quy mô (VRS) đề xuất bởi Charnes, Cooper, và Rhodes (1978).

Theo Charnes và cộng sự (1978), mô hình DEA - CSR được đề xuất như sau: Giả sử với n đơn vị có $\mathrm{k}$ yếu tố đầu vào và $\mathrm{m}$ yếu tố đầu ra, gọi vectơ $\mathrm{V}_{\mathrm{i}}, \mathrm{u}_{\mathrm{i}}$ lần lượt là tập hợp đầu vào và đầu ra tương ứng. Hiệu quả kỹ thuật của từng DMU được tính như sau:

$$
\operatorname{Maximize} \frac{\sum_{r=1}^{S} u_{r} y_{r k}}{\sum_{i=1}^{m} v_{i} x_{i k}}
$$

Điều kiện:

$$
\begin{array}{ll}
\frac{\sum_{r=1}^{S} u_{r} y_{r k}}{\sum_{i=1}^{m} v_{i} x_{i k}} \leq 1 & \mathrm{j}=1, \ldots, \mathrm{n} \\
\mathrm{u}_{\mathrm{r}}, \mathrm{v}_{\mathrm{i}}>0 & \mathrm{r}=1, \ldots \mathrm{s} ; \mathrm{i}=1, \ldots, \mathrm{m}
\end{array}
$$

Bài toán quy hoạch tuyến tính này có thể giải quyết theo hai hướng: Mô hình định hướng đầu vào và mô hình định hướng đầu ra.

$$
\text { Maximize } \sum_{r=1}^{S} u_{r} y_{r k}
$$

Điều kiện:

$$
\begin{array}{lr}
\sum_{i=1}^{m} v_{i} x_{i j}-\sum_{r=1}^{s} u_{r} y_{r j} \geq 0 \mathrm{j}=1, \ldots, \mathrm{n} & \\
\sum_{i=1}^{m} v_{i} x_{i k}=1 & \mathrm{r}=1, \ldots, \mathrm{s} ; \mathrm{i}=1, \ldots, \mathrm{m}
\end{array}
$$

Để phù hợp trong tính toán, các nhà nghiên cứu đã đưa ra cách tính đối ngẫu trong chương trình tuyến tính để giảm bớt số điều kiện ràng buộc của mô hình từ $\mathrm{n}+1$ xuống $\mathrm{s}+\mathrm{m}$. Mô hình cuối cùng có dạng:

\section{Minimize $\Theta_{\mathrm{k}}$}

Điều kiện:

$$
\begin{array}{lr}
\mathrm{y}_{\mathrm{rk}}-\sum_{j=1}^{n} \lambda_{j} y_{r j} \leq 0 & \mathrm{r}=1, \ldots, \mathrm{s} \\
\Theta_{\mathrm{k}} \mathrm{x}_{\mathrm{ik}}-\sum_{j=1}^{n} \lambda_{j} y_{r j} \geq 0 & \mathrm{i}=1, \ldots, \mathrm{m} \\
\lambda_{\mathrm{j}} \geq 0 & \mathrm{j}=1, \ldots, \mathrm{n}
\end{array}
$$

Trong đó: $\Theta_{\mathrm{k}}$ là đại lượng vô hướng thể hiện mức độ hiệu quả của ngân hàng và ג là vectơ hằng số các yếu tố đầu vào, đầu ra.

Nếu $\Theta=1$ : Ngân hàng đạt hiệu quả

$\Theta<1$ : Ngân hàng không đạt hiệu quả

Mô hình DEA - VSR được thành lập dựa trên mô hình DEA - CSR nhưng được bổ sung thêm ràng buộc tổng các trọng số phải bằng 1 hoặc mô hình hiệu suất giảm dần (tăng dần) theo quy mô là tổng các trọng số nhỏ hơn hoặc bằng 1 .

- Bước 2: Kiểm định các nhân tố tác động đến hiệu quả kỹ thuật các NHTMCP

Mô hình kiểm định các nhân tố tác động đến hiệu quả kỹ thuật NHTMCP và các biến sử dụng trong mô hình được đưa ra dựa trên khung tiêu chuẩn CAMELS và các nghiên cứu trước được đề xuất như sau:

$$
\mathrm{TE}_{\mathrm{it}}=\beta_{0}+\beta_{1} \mathrm{BANKSIZE}_{\mathrm{it}}+\beta_{2} \mathrm{ROA}_{\mathrm{it}}+\beta_{3} \mathrm{NPL}_{\mathrm{it}}+\beta_{4} \mathrm{DLR}_{\mathrm{it}}+\beta_{5} \mathrm{ETA}_{\mathrm{it}}+\varepsilon_{\mathrm{it}}
$$




\section{Bảng 2}

Các biến sử dụng trong mô hình hồi quy

\begin{tabular}{|c|c|c|}
\hline Biến & Ký hiệu & Cách đo lường \\
\hline \multicolumn{3}{|l|}{ Biến phu thuộc } \\
\hline Hiệu quả kỹ thuật & $\mathrm{TE}$ & $\begin{array}{l}\text { Hiệu quả kỹ thuật ngân hàng i được ước lượng bằng } \\
\text { phương pháp DEA hoặc SFA }\end{array}$ \\
\hline \multicolumn{3}{|l|}{ Các biến độc lập } \\
\hline Quy mô ngân hàng & BANKSIZE & Logarit (tổng tài sản) \\
\hline Tỷ số lợi nhuận/ tổng tài sản & ROA & Lợi nhuận/tổng tài sản \\
\hline Tỷ lệ nợ xấu/tổng dư nợ & NPL & Nợ xấu/tổng dư nợ \\
\hline Tỷ lệ tiền gửi/cho vay & DLR & Tiền gửi/cho vay \\
\hline Tỷ lệ vốn chủ sở hữu/tổng tài sản & ETA & Vốn chủ sở hữu/tổng tài sản \\
\hline
\end{tabular}

Nguồn: Kết quả phân tích dữ liệu của nhóm nghiên cứu

\subsection{Dũ liệu và phương pháp nghiên cúu}

Theo báo cáo của Ngân hàng Nhà nước tại thời điểm 30/06/2019, có 30 NHTMCP tại Việt Nam đang hoạt động. Tuy nhiên, do đặc thù về công bố thông tin hoạt động kinh doanh và một số ngân hàng được thành lập và hợp nhất trong khoảng thời gian nghiên cứu từ giai đoạn 2011 đến 2018 và để liên tục mẫu dữ liệu nghiên cứu sẽ bao gồm 19 với dữ liệu được thu thập từ các báo cáo tài chính năm đã qua kiểm toán, báo cáo thường niên, website thông tin của 19 NHTMCP Việt Nam trong giai đoạn 2011 - 2018.

Nghiên cứu sử dụng kết hợp phương pháp phân tích biên ngẫu nhiên (SFA), phương pháp phân tích bao dữ liệu (DEA) để tính toán hiệu quả kỹ thuật của các NHTMCP Việt Nam. Phương pháp bình phương tối thiểu gộp (Pooled OLS), mô hình tác động ngẫu nhiên (REM) và mô hình tác động cố định (FEM) cũng lần lượt được sử dụng để tìm ra mô hình phù hợp nhất với mẫu dữ liệu nghiên cứu nhằm xác định các nhân tố tác động đến hiệu quả kỹ thuật ngân hàng. Danh sách các ngân hàng trong mẫu nghiên cứu được tổng hợp trong Bảng 3.

\section{Bảng 3}

Tổng hợp các ngân hàng trong mẫu nghiên cứu

\begin{tabular}{|c|l|c|}
\hline STT & \multicolumn{1}{|c|}{ Tên NHTMCP } & Mã chứng khoán \\
\hline 1 & Ngân hàng Á Châu & ACB \\
\hline 2 & Ngân hàng Bắc Á & BAB \\
\hline 3 & Ngân hàng Đầu tư và Phát triển Việt Nam & BID \\
\hline 4 & Ngân hàng Công thương Việt Nam & CTG \\
\hline 5 & Ngân hàng Xuất Nhập Khẩu Việt Nam & EIB \\
\hline 6 & Ngân hàng Phát triển TP.HCM & HDB \\
\hline 7 & Ngân hàng Kiên Long & KLB \\
\hline 8 & Ngân hàng Bưu điện Liên Việt & LPB \\
\hline 9 & Ngân hàng Quân Đội & MBB \\
\hline
\end{tabular}




\begin{tabular}{|c|l|c|}
\hline STT & \multicolumn{1}{|c|}{ Tên NHTMCP } & Mã chứng khoán \\
\hline 10 & Ngân hàng Hàng Hải & MSB \\
\hline 11 & Ngân hàng Nam Á & NAB \\
\hline 12 & Ngân hàng Quốc dân & NCB \\
\hline 13 & Ngân hàng Xăng dầu Petrolimex & PGB \\
\hline 14 & Ngân hàng Sài Gòn - Hà Nội & SHB \\
\hline 15 & Ngân hàng Sài Gòn Thương Tín & STB \\
\hline 16 & Ngân hàng Kỹ Thương & TCB \\
\hline 17 & Ngân hàng Tiên Phong & TPB \\
\hline 18 & Ngân hàng Ngoại Thương Việt Nam & VCB \\
\hline 19 & Ngân hàng Việt Nam Thịnh Vượng & VPB \\
\hline
\end{tabular}

Nguồn: Kết quả phân tích dữ liệu của nhóm nghiên cứu

\section{Kết quả nghiên cứu}

\subsection{Kết quả ước luọng hiệu quả kỹ thuật các Ngân hàng thuơng mại cổ phần tại Việt Nam}

4.1.1. Kết quả ước lương hiệu quả kỹ thuật bằng phwơng pháp SFA

Trước hết, nghiên cứu thực hiện kiểm định dạng hàm sản xuất và kiểm định có hay không phi hiệu quả kỹ thuật.

Kết quả kiểm định được trình bày ở Bảng 4 , trong đó giá trị tới hạn ở mức ý nghĩa $1 \%$ và $5 \%$ được tra trong bảng từ nghiên cứu của Kodde và Palm (1986), chi tiết như sau:

\section{Bảng 4}

Kiểm định tỷ số hợp lý tổng quát cho tham số của mô hình hàm sản xuất biên ngẫu nhiên (SFA)

\begin{tabular}{|l|c|c|c|c|}
\hline \multirow{2}{*}{ Giả thuyết $\mathbf{H}_{\mathbf{0}}$} & \multirow{2}{*}{ Giá trị LR statistic } & \multicolumn{2}{|c|}{ Giá trị tới hạn } & \multirow{2}{*}{ Quyết định } \\
\cline { 3 - 4 } & $\mathbf{1 \%}$ & $\mathbf{5 \%}$ & \\
\hline 1. Lựa chọn dạng hàm giữa Cobb-Douglas và Translog (bậc tự do df = 6) \\
\hline $\mathrm{H}_{0:}: \beta_{1=} \beta_{2}=\beta_{3}=\lambda_{1}=\lambda_{2}=\lambda_{3}=0$ & 50,602 & 16,074 & 11,911 & \multirow{2}{*}{ Bác bỏ $\mathrm{H}_{0}$} \\
\hline 2. Xác định phi HQKT (bậc tự do df = 3) & $-2,000$ & 10,501 & 7,045 & Không bác bỏ \\
\hline $\mathrm{H}_{0}:$ Không tồn tại phi HQKT & 5,906 & 5,412 & 2,706 & Bác bỏ $\mathrm{H}_{0}$ \\
\hline 3. Phi HQKT không thay đổi theo thời gian (bậc tự do df $=1)$ \\
\hline $\mathrm{H}_{0}: \eta=0$
\end{tabular}

Nguồn: Kết quả xử lý từ dữ liệu điều tra

Như vậy, mô hình nghiên cứu xem xét là mô hình hàm sản xuất Translog và không tồn tại phi hiệu quả kỹ thuật. Dựa trên kết quả này, mô hình hàm sản xuất biên được ước lượng như sau: 


\section{Bảng 5}

Hiệu quả kỹ thuật đo lường bằng phương pháp SFA

\begin{tabular}{|l|c|c|c|c|c|c|c|c|}
\hline \multicolumn{1}{|c|}{ Năm } & $\mathbf{2 0 1 1}$ & $\mathbf{2 0 1 2}$ & $\mathbf{2 0 1 3}$ & $\mathbf{2 0 1 4}$ & $\mathbf{2 0 1 5}$ & $\mathbf{2 0 1 6}$ & $\mathbf{2 0 1 7}$ & $\mathbf{2 0 1 8}$ \\
\hline Giá trị trung bình & 0,766 & 0,728 & 0,701 & 0,666 & 0,664 & 0,617 & 0,649 & 0,642 \\
\hline Độ lệch chuẩn & 0,129 & 0,127 & 0,140 & 0,111 & 0,132 & 0,173 & 0,146 & 0,138 \\
\hline Giá trị nhỏ nhất & 0,346 & 0,387 & 0,314 & 0,360 & 0,398 & 0,261 & 0,387 & 0,386 \\
\hline Giá trị lớn nhất & 0,929 & 0,888 & 0,850 & 0,895 & 0,836 & 0,902 & 0,870 & 0,893 \\
\hline
\end{tabular}

Nguồn: Kết quả xử lý từ dữ liệu điều tra

Dựa trên kết quả đo lường hiệu quả kỹ thuật của các NHTMCP tại Việt Nam bằng phương pháp SFA tại Bảng 5, giá trị hiệu quả kỹ thuật bình quân của các NHTMCP trong giai đoạn 2011 - 2018 có xu hướng giảm dần, đặc biệt từ năm 2012 đến năm 2016. Đây là thời kỳ tái cấu trúc hệ thống ngân hàng. Trong đó, năm 2016 là năm có mức hiệu quả kỹ thuật bình quân thấp nhất trong thời gian nghiên cứu $(0,617)$, là năm Việt Nam chịu nhiều tác động tiêu cực như: Hạn hán, thiên tai và kinh tế toàn cầu tăng trưởng chậm hơn so dự báo. Mặt bằng lãi suất huy động tăng nhẹ từ $0,20-0,40 \%$ do việc đẩy mạnh huy động tiền gửi, trong khi NHNN vẫn duy trì định hướng lãi suất cho vay thấp để hỗ trợ tăng trưởng kinh tế. Điều này đã ảnh hưởng không nhỏ đến hoạt động tín dụng của các ngân hàng, dẫn đến lãi suất cho vay chỉ được giảm ở mức cầm chừng, không tạo được sức lan tỏa toàn thị trường. Bên cạnh đó, việc áp dụng thí điểm chuẩn Basel II tại 10 NHTM thí điểm (BIDV, Vietinbank, Vietcombank, Techcombank, ACB, VPBank, MB, Sacombank, MaritimeBank, VIB) gây ra áp lực tăng vốn và chi phí hoạt động cho các ngân hàng vì phần lớn các ngân hàng tại Việt Nam đều dựa vào nguồn vốn cấp hai để cải thiện tình hình kinh doanh trong ngắn hạn. Tuy nhiên, đến năm 2017 và 2018 tín hiệu tích cực đã xuất hiện trở lại với mức hiệu quả kỹ thuật bình quân đã dần được cải thiện (năm 2017 giá trị trung bình đạt 0,649 tương đương tăng $5,19 \%$ so với năm 2016; năm 2018 giá trị trung bình đạt 0,642 tương đương tăng 4,05\% so với năm 2016), cho thấy sự thành công của NHNN trong việc điều hành tỷ giá, tín dụng và kiểm soát nợ xấu và đặc biệt có sáu ngân hàng đầu tiên đã tuyên bố thành công trong việc áp dụng hệ thống Basel II trong hoạt động kinh doanh của mình (VPBank, MBBank, TPBank, Vietcombank, VIB, $\mathrm{OCB})$, dự báo tín hiệu khả quan cho hoạt động ngân hàng vào năm 2019 và những năm sắp tới.

\subsubsection{Kết quả ước lượng hiệu quả kỹ thuật bằng phioong pháp DEA}

Kết quả thống kê hiệu quả kỹ thuật qua các năm được trình bày tóm tắt ở Bảng 6 :

\section{Bảng 6}

Hiệu quả kỹ thuật đo lường bằng phương pháp DEA

\begin{tabular}{|l|c|c|c|c|c|c|c|c|}
\hline \multicolumn{1}{|c|}{ Năm } & $\mathbf{2 0 1 1}$ & $\mathbf{2 0 1 2}$ & $\mathbf{2 0 1 3}$ & $\mathbf{2 0 1 4}$ & $\mathbf{2 0 1 5}$ & $\mathbf{2 0 1 6}$ & $\mathbf{2 0 1 7}$ & $\mathbf{2 0 1 8}$ \\
\hline Giá trị trung bình & 0,961 & 0,969 & 0,980 & 0,982 & 0,981 & 0,990 & 0,911 & 0,964 \\
\hline Độ lệch chuẩn & 0,038 & 0,024 & 0,023 & 0,018 & 0,019 & 0,013 & 0,063 & 0,031 \\
\hline Giá trị nhỏ nhất & 0,901 & 0,943 & 0,941 & 0,954 & 0,944 & 0,962 & 0,840 & 0,911 \\
\hline Giá trị lớn nhất & 1,000 & 1,000 & 1,000 & 1,000 & 1,000 & 1,000 & 1,000 & 1,000 \\
\hline
\end{tabular}

Nguồn: Kết quả xử lý từ dữ liệu điều tra

Nhìn chung, kết quả đo lường hiệu quả kỹ thuật của các NHTMCP đo bằng phương pháp DEA với giả định VRS có kết quả tương tự với phương pháp SFA. Mức hiệu quả bình quân của 
các ngân hàng đạt tương đối tốt (đạt trên 50\%) và có xu hướng giảm dần từ năm 2011 - 2016. Năm 2017 và 2018 có dấu hiệu tăng trưởng trở lại (mức hiệu quả kỹ thuật năm 2017 trung bình đạt 0,911; năm 2018 đạt 0,964, tương đương tăng 5,82\% so với năm 2017).

Kết quả ước lượng chỉ số Malmquist bình quân cho toàn bộ mẫu, từng ngân hàng trong giai đoạn 2011 - 2018 được trình bày tóm tắt trong Bảng 7 và Bảng 8 :

\section{Bảng 7}

Chỉ số Malmquist bình quân thời kỳ 2011 - 2018

\begin{tabular}{|c|c|c|c|c|c|c|}
\hline Năm & effch & techch & pech & sech & tfpch & Số quan sát \\
\hline $2011-2012$ & 1,387 & 1,709 & 1,008 & 1,376 & 2,370 & 19 \\
\hline $2012-2013$ & 1,041 & 1,647 & 1,012 & 1,029 & 1,715 & 19 \\
\hline $2013-2014$ & 1,079 & 1,244 & 1,002 & 1,077 & 1,343 & 19 \\
\hline $2014-2015$ & 1,052 & 1,257 & 0,999 & 1,054 & 1,323 & 19 \\
\hline $2015-2016$ & 1,050 & 1,142 & 1,010 & 1,039 & 1,199 & 19 \\
\hline $2016-2017$ & 0,891 & 1,310 & 0,918 & 0,971 & 1,168 & 19 \\
\hline $2017-2018$ & 1,902 & 1,034 & 1,059 & 1,031 & 1,130 & 19 \\
\hline
\end{tabular}

Trong đó: effch: Thay đổi hiệu quả kỹ thuật; techch: Thay đổi tiến độ công nghệ; pech: Thay đổi hiệu quả thuần; sech: Thay đổi hiệu quả quy mô; tfpch: Thay đổi năng suất nhân tố tổng hợp.

Nguồn: Kết quả xử lý từ dữ liệu điều tra

Bảng 7 cho thấy sự thay đổi của năng suất nhân tố tổng hợp trong thời kỳ nghiên cứu đều lớn hơn 1 , trung bình đạt 1,455 (cụ thể: thời kỳ 2011 - 2012 đạt 2,370; 2012 - 2013 đạt 1,715; 2013 - 2014 đạt 1,343; 2014 - 2015 dạt 1,323; 2015 - 2016 đạt 1,199; 2016 - 2017 đạt 1,168; 2017 2018 đạt 1,130). Nguyên nhân đến từ sự thay đổi tiến độ công nghệ, thay đổi hiệu quả thuần và hiệu quả quy mô qua các năm, đặc biệt là thay đổi tiến bộ công nghệ luôn lớn hơn 1 qua các năm. Điều này cho thấy các ngân hàng đang chú trọng rất nhiều vào việc đầu tư, áp dụng công nghệ tiên tiến trong hoạt động kinh doanh của mình thay vì sử dụng nhiều lao động.

Bảng 8 dưới đây trình bày kết quả ước lượng thay đổi hiệu quả kỹ thuật, thay đổi tiến bộ công nghệ, thay đổi hiệu quả theo quy mô và thay đổi năng suất nhân tố tổng hợp của 19 NHTMCP trong giai đoạn nghiên cứu $2011-2018$.

\section{Bảng 8}

Kết quả ước lượng effch, techch, pech, sech, tfpch cho 19 NHTMCP tại Việt Nam giai đoạn 2011 $-2018$

\begin{tabular}{|c|c|c|c|c|c|c|}
\hline STT & Ngân hàng & effch & techch & pech & sech & tfpch \\
\hline 1 & ACB & 1,151 & 1,313 & 1,000 & 1,151 & 1,511 \\
\hline 2 & BAB & 1,149 & 1,306 & 1,009 & 1,139 & 1,501 \\
\hline 3 & BID & 1,149 & 1,312 & 1,007 & 1,141 & 1,507 \\
\hline 4 & CTG & 1,157 & 1,319 & 1,012 & 1,144 & 1,527 \\
\hline 5 & EIB & 1,148 & 1,312 & 1,003 & 1,144 & 1,506 \\
\hline 6 & HDB & 1,148 & 1,306 & 1,004 & 1,144 & 1,500 \\
\hline 7 & KLB & 1,149 & 1,312 & 1,005 & 1,143 & 1,507 \\
\hline
\end{tabular}




\begin{tabular}{|c|c|c|c|c|c|c|}
\hline STT & Ngân hàng & effch & techch & pech & sech & tfpch \\
\hline 8 & LPB & 1,148 & 1,310 & 1,004 & 1,143 & 1,505 \\
\hline 9 & MBB & 1,024 & 1,316 & 0,989 & 1,035 & 1,347 \\
\hline 10 & MSB & 1,023 & 1,313 & 0,988 & 1,035 & 1,344 \\
\hline 11 & NAB & 1,024 & 1,323 & 0,989 & 1,035 & 1,354 \\
\hline 12 & NCB & 1,045 & 1,330 & 1,001 & 1,043 & 1,389 \\
\hline 13 & PGB & 1,043 & 1,320 & 1,000 & 1,043 & 1,378 \\
\hline 14 & SHB & 1,048 & 1,317 & 1,004 & 1,044 & 1,381 \\
\hline 15 & STB & 1,047 & 1,317 & 1,003 & 1,044 & 1,379 \\
\hline 16 & TCB & 1,048 & 1,315 & 1,004 & 1,044 & 1,379 \\
\hline 17 & TPB & 0,997 & 1,317 & 0,997 & 1,000 & 1,314 \\
\hline 18 & VCB & 0,996 & 1,313 & 0,996 & 1,000 & 1,307 \\
\hline 19 & VPB & 0,993 & 1,322 & 0,993 & 1,000 & 1,313 \\
\hline \multicolumn{2}{|r|}{ Trung bình $2011-2018$} & 1,076 & 1,315 & 1,000 & 1,076 & 1,416 \\
\hline
\end{tabular}

Nguồn: Kết quả xử lý từ dữ liệu điều tra

Nhìn chung, 08 ngân hàng có kết quả thay đổi nhân tố tổng hợp cao nhất trong giai đoạn mẫu nghiên cứu là $\mathrm{ACB}, \mathrm{BAB}, \mathrm{BID}, \mathrm{CTG}, \mathrm{EIB}, \mathrm{HDB}, \mathrm{KLB}$ và $\mathrm{LPB}$. Điều này chủ yếu đến từ việc thay đổi mạnh tiến bộ công nghệ trong hoạt động của các ngân hàng này.

\subsection{3. Đánh giá SFA - DEA}

Bảng 9 và Bảng 10 lần lượt trình bày mức hiệu quả kỹ thuật của các NHTMCP theo hai phương pháp SFA, DEA và kết quả xếp hạng hiệu quả kỹ thuật của các ngân hàng nghiên cứu theo hai phương pháp. Nhìn chung, SFA và DEA cho kết quả khác nhau về chỉ số hiệu quả kỹ thuật nhưng nếu xếp hạng về mức độ hiệu quả của các NHTMCP thì có kết quả tương đồng. Chẳng hạn đối với phương pháp $S F A$, ngân hàng $\mathrm{VPB}$ và $\mathrm{TCB}$ lần lượt là hai ngân hàng đạt mức hiệu quả kỹ thuật cao nhất (VPB: 0,790 ; TCB: 0,754$)$, STB có mức hiệu quả kỹ thuật thấp nhất $(0,513)$. Đối với phương pháp $\mathrm{DEA}$, ngân hàng $\mathrm{ACB}, \mathrm{MBB}, \mathrm{TPB}$ và $\mathrm{VCB}$ là những ngân hàng đạt hiệu quả với mức hiệu quả kỹ thuật đạt được là 1,000 ; LPB là ngân hàng có mức hiệu quả thấp nhất trong giai đoạn nghiên cứu (hiệu quả kỹ thuật đạt được là 0,901).

\section{Bảng 9}

Kết quả hiệu quả kỹ thuật của các NHTMCP Việt Nam giai đoạn 2011 - 2018 theo phương pháp SFA và DEA

\begin{tabular}{|c|c|c|c|}
\hline STT & Ngân hàng & SFA_TE & DEA_TE \\
\hline 1 & ACB & 0,706 & 1,000 \\
\hline 2 & BAB & 0,679 & 0,926 \\
\hline 3 & BID & 0,752 & 0,924 \\
\hline 4 & CTG & 0,642 & 0,922 \\
\hline 5 & EIB & 0,605 & 0,921 \\
\hline 6 & HDB & 0,643 & 0,915 \\
\hline 7 & KLB & 0,670 & 0,903 \\
\hline 8 & LPB & 0,715 & 0,901 \\
\hline
\end{tabular}




\begin{tabular}{|c|c|c|c|}
\hline STT & Ngân hàng & SFA_TE & DEA_TE \\
\hline 9 & MBB & 0,748 & 1,000 \\
\hline 10 & MSB & 0,708 & 0,999 \\
\hline 11 & NAB & 0,676 & 0,987 \\
\hline 12 & NCB & 0,556 & 0,990 \\
\hline 13 & PGB & 0,711 & 0,994 \\
\hline 14 & SHB & 0,645 & 0,971 \\
\hline 15 & STB & 0,513 & 0,962 \\
\hline 16 & TCB & 0,754 & 0,958 \\
\hline 17 & TPB & 0,640 & 1,000 \\
\hline 18 & VCB & 0,739 & 1,000 \\
\hline 19 & VPB & 0,790 & 0,990 \\
\hline \multicolumn{2}{|c|}{ Trung bình $2011-2018$} & 0,677 & 0,961 \\
\hline
\end{tabular}

Nguồn: Kết quả xử lý từ dữ liệu điều tra

\section{Bảng 10}

Xếp hạng hiệu quả kỹ thuật của các NHTMCP Việt Nam giai đoạn 2011 - 2018 theo phương pháp SFA và DEA

\begin{tabular}{|c|c|c|}
\hline Ngân hàng & SFA_TE & DEA_TE \\
\hline $\mathrm{ACB}$ & 9 & 1 \\
\hline $\mathrm{BAB}$ & 10 & 13 \\
\hline BID & 3 & 14 \\
\hline CTG & 15 & 15 \\
\hline EIB & 17 & 16 \\
\hline HDB & 14 & 17 \\
\hline KLB & 12 & 18 \\
\hline LPB & 6 & 19 \\
\hline MBB & 4 & 1 \\
\hline MSB & 8 & 5 \\
\hline NAB & 11 & 9 \\
\hline $\mathrm{NCB}$ & 18 & 7 \\
\hline PGB & 7 & 6 \\
\hline SHB & 13 & 10 \\
\hline STB & 19 & 11 \\
\hline TCB & 2 & 12 \\
\hline TPB & 16 & 1 \\
\hline VCB & 5 & 1 \\
\hline VPB & 1 & 7 \\
\hline
\end{tabular}

Nguồn: Kết quả xử lý từ dữ liệu điều tra 


\subsection{Phân tích các nhân tố tác động đến hiệu quả họ̣t động các Ngân hàng thuơng mại cổ phần tại Việt Nam}

\subsubsection{Phân tích hệ số tương quan}

Bảng 11 thể hiện ma trận tương quan giữa các biến trong mô hình. Kết quả này cho thấy mối tương quan giữa các biến trong mô hình là thấp. Đặc biệt giữa các biến NPL, ROA, DLR và ETA là hầu như không xuất hiện mối tương quan.

\section{Bảng 11}

Ma trận tương quan

\begin{tabular}{|l|c|c|c|c|c|}
\hline & LnAssets & ROA & NPL & DLR & ETA \\
\hline LnAssets & 1,000 & & & & \\
\hline ROA & 0,171 & 1,000 & & & \\
\hline NPL & $-0,212$ & $-0,066$ & 1,000 & & \\
\hline DLR & $-0,055$ & $-0,244$ & $-0,063$ & 1,000 & \\
\hline ETA & $-0,648$ & 0,219 & 0,293 & $-0,068$ & 1,000 \\
\hline
\end{tabular}

Nguồn: Kết quả xử lý từ dữ liệu điều tra

\subsubsection{Kết quả phân tích hồi quy}

Nghiên cứu tiến hành hồi quy các nhân tố ảnh hưởng đến hiệu quả kỹ thuật lần lượt với các phương pháp Pooled OLS, FEM, REM.

\section{Bảng 12}

Kết quả hồi quy lần thứ nhất với biến phụ thuộc là TE_SFA

\begin{tabular}{lcccccc}
\multicolumn{1}{c}{ Tên biến } & \multicolumn{2}{c}{ Pooled OLS } & \multicolumn{2}{c}{ FEM } & \multicolumn{2}{c}{ REM } \\
& Hệ số & P-value & Hệ số & P-value & Hệ số & P-value \\
Cons & 0,355 & 0,445 & 3,514 & 1,048 & 0,683 & 0,584 \\
LnAssets & 0,006 & 0,644 & $-0,089$ & $-0,031$ & $-0,004$ & 0,017 \\
ROA & 7,892 & 0,000 & 7,521 & 1,621 & 7,215 & 1,585 \\
NPL & $-0,172$ & 0,834 & $-1,084$ & 0,861 & $-0,461$ & $-0,827$ \\
DLR & $-0,307$ & 0,826 & $-1,493$ & 1,366 & $-0,854$ & 1,359 \\
ETA & 0,810 & 0,079 & 0,644 & 0,531 & 1,026 & 0,475 \\
Prob>F & \multicolumn{0}{c}{0,000} & & 0,000 & & 0,000 \\
R-squared & 0,241 & & 0,075 &
\end{tabular}

Nguồn: Kết quả xử lý từ dữ liệu điều tra 


\section{Bảng 13}

Kết quả hồi quy lần thứ nhất với biến phụ thuộc là TE_DEA

\begin{tabular}{|c|c|c|c|c|c|c|}
\hline \multirow{2}{*}{ Tên biến } & \multicolumn{2}{|c|}{ Pooled OLS } & \multicolumn{2}{|c|}{ FEM } & \multicolumn{2}{|c|}{ REM } \\
\hline & Hệ số & $\mathrm{P}$-value & Hệ số & P-value & Hệ số & P-value \\
\hline Cons & 0,896 & 0,000 & 1,499 & 0,364 & 0,896 & 0,145 \\
\hline LnAssets & 0,002 & 0,677 & $-0,016$ & 0,011 & 0,002 & 0,004 \\
\hline ROA & $-0,462$ & 0,360 & $-0,681$ & 0,564 & $-0,462$ & 0,503 \\
\hline NPL & 0,207 & 0,420 & 0,014 & 0,300 & 0,207 & 0,255 \\
\hline DLR & 0,358 & 0,412 & $-0,087$ & 0,475 & 0,358 & 0,436 \\
\hline ETA & 0,086 & 0,550 & $-0,040$ & 0,185 & 0,086 & 0,143 \\
\hline Prob $>F$ & & \multicolumn{2}{|c|}{0,702} & \multicolumn{2}{|c|}{0,265} & 0,702 \\
\hline R-squared & & \multicolumn{2}{|l|}{0,020} & \multicolumn{2}{|c|}{0,002} & 0,020 \\
\hline \multicolumn{7}{|c|}{ Nguồn: Kết quả xử lý từ dữ liệu điều tra } \\
\hline \multicolumn{7}{|c|}{$\begin{array}{l}\text { Để lựa chọn mô hình phù hợp, nghiên cứu lần lượt tiến hành kiểm định Hausman, } \mathrm{F} \text {, } \\
\text { Lagrange, kêt quả được trình bày lần lượt tại Bảng } 14,15 \text {, và } 16 \text {. Như vậy, dựa vào kết quả cho } \\
\text { thấy mô hình ước lượng FEM là phù hợp để kiểm định với biến phụ thuộc là TE_SFA và mô hình } \\
\text { ước lượng Pooled OLS là phù hợp để kiểm định với biến phụ thuộc là TE_DEA. }\end{array}$} \\
\hline \multicolumn{7}{|l|}{ Bảng 14} \\
\hline \multicolumn{7}{|c|}{ Kết quả kiểm định Hausman } \\
\hline \multicolumn{2}{|c|}{ Biến phụ thuộc } & & \multicolumn{2}{|c|}{ Chi2 (5) } & \multicolumn{2}{|c|}{ Prob > chi2 } \\
\hline TE_DEA & & & & 9,90 & & 0,078 \\
\hline TE_SFA & & & & 23,95 & & 0,000 \\
\hline
\end{tabular}

Nguồn: Kết quả xử lý từ dữ liệu điều tra

\section{Bảng 15}

Kiểm định $\mathrm{F}$

\begin{tabular}{|c|r|r|}
\hline Biến phụ thuộc & F (5, 128) & \multicolumn{2}{|c|}{ Prob > F } \\
\hline TE_SFA & 12,13 & 0,000 \\
\hline
\end{tabular}

Nguồn: Kết quả xử lý từ dữ liệu điều tra

\section{Bảng 16}

Kiểm định Breusch - Pagan Lagrange Multiplier

\begin{tabular}{|l|r|r|}
\hline \multicolumn{1}{|c|}{ Biến phụ thuộc } & Chibar2 (01) & \multicolumn{2}{|c|}{ Prob > chibar2 } \\
\hline TE_DEA & 0,00 & 1,000 \\
\hline
\end{tabular}

Nguồn: Kết quả xử lý từ dữ liệu điều tra

Tiếp theo, nghiên cứu tiến hành kiểm tra sai phạm trước khi đưa ra kết quả hồi quy cuối cùng. Với kết quả từ Bảng 17 cho thấy đối với biến phụ thuộc TE_SFA, mô hình gặp hiện tượng phương sai sai số thay đổi. Còn đối với biến phụ thuộc là TE_DEA, mô hình gặp cả hiện tượng phương sai sai số thay đổi và tự tương quan của sai số giữa các đơn vị chéo. Do vậy, bài viết sẽ thực hiện hồi quy bằng quy bằng phương pháp GLS để khắc phục khuyết tật này. 


\section{Bảng 17}

Kết quả kiểm định đa cộng tuyến, hiện tượng phương sai thay đổi, tự tương quan

Kiểm định

Đa cộng tuyến

Phương sai thay đổi

Tự tương quan
Kết quả

$\mathrm{VIF}=1,59<10$

TE_SFA: Prob $>$ chi $2=0,000$

TE_DEA: Prob $>$ chi $2=0,032$

TE_SFA: Prob $>$ chi $2=0,967$

TE_DEA: Prob $>$ chi $2=0,002$

\section{Kết luận}

Không có hiện tượng đa cộng tuyến Vi phạm

Vi phạm

Không vi phạm

Vi phạm

Nguồn: Kết quả xử lý từ dữ liệu điều tra

Kết quả ước lượng Pooled OLS đối với biến phụ thuộc là TE_DEA ở Bảng 18 và FEM đối với biến phụ thuộc là TE_SFA được trình bày ở Bảng 19. Cả hai mô hình đều có giá trị p-value đều là 0,000 chứng tỏ các hệ số hồi quy đồng thời bằng không đều bị bác bỏ ở mức ý nghĩa $5 \%$. Nghĩa là mô hình trên phù hợp với nghiên cứu.

\section{Bảng 18}

Kết quả ước lượng hồi quy lần hai với biến phụ thuộc là TE_SFA

\begin{tabular}{|l|c|c|c|c|c|c|}
\hline \multicolumn{1}{|c|}{ TE_SFA } & Hệ số & Sai số chuẩn & $\mathbf{z}$ & $\mathbf{P}>|\mathbf{z}|$ & \multicolumn{2}{c|}{ Độ tin cậy 95\% } \\
\hline Hệ số chặn & 0,090 & 0,377 & 0,24 & 0,811 & $-0,649$ & 0,829 \\
\hline LnAssets & 0,014 & 0,011 & 1,28 & 0,202 & $-0,008$ & 0,036 \\
\hline ROA & $8,459^{* * *}$ & 1,391 & 6,08 & 0,000 & 5,732 & 11,186 \\
\hline NPL & 0,766 & 0,615 & 1,24 & 0,213 & $-0,440$ & 1,972 \\
\hline DLR & $-0,746$ & 1,062 & $-0,70$ & 0,483 & $-2,828$ & 1,336 \\
\hline ETA & $0,810^{* *}$ & 0,366 & 2,21 & 0,027 & 0,093 & 1,527 \\
\hline Prob > chi2 & 0,000 & \multicolumn{7}{|l|}{} \\
\hline Wald chi2 (5) & 82,10 &
\end{tabular}

Nguồn: Kết quả xử lý từ dữ liệu điều tra

\section{Bảng 19}

Kết quả ước lượng hồi quy lần hai với biến phụ thuộc là TE_DEA

\begin{tabular}{|l|c|c|c|c|c|c|}
\hline \multicolumn{1}{|c|}{ TE_DEA } & Hệ số & Sai số chuẩn & $\mathbf{z}$ & \multicolumn{1}{c|}{ P>|z| } & \multicolumn{2}{c|}{ Độ tin cậy 95\% } \\
\hline Hệ số chặn & $0,834^{* * *}$ & 0,087 & 0,54 & 0,000 & 0,663 & 1,005 \\
\hline LnAssets & 0,003 & 0,002 & 1,40 & 0,163 & $-0,001$ & 0,008 \\
\hline ROA & $-0,568$ & 0,406 & $-1,40$ & 0,162 & $-1,362$ & 0,227 \\
\hline NPL & $0,430 * *$ & 0,201 & 2,14 & 0,032 & 0,037 & 0,823 \\
\hline DLR & 0,600 & 0,749 & 0,80 & 0,423 & $-0,867$ & 2,068 \\
\hline ETA & $0,136^{*}$ & 0,071 & 1,92 & 0,055 & $-0,003$ & 0,274 \\
\hline Prob > chi2 & 0,000 & \multicolumn{7}{|l}{} \\
\hline Wald chi2 (5) & 38,56 &
\end{tabular}

Ghi chú: *, ** và *** lần lượt là các mức ý nghĩa thống kê $10 \%, 5 \%$ và $1 \%$

Nguồn: Kết quả xử lý từ dữ liệu điều tra 


\subsubsection{Thảo luận kết quả nghiên cưu}

Kết quả phân tích cho thấy các yếu tố thuộc nhóm hiệu quả quản lý như ROA, ETA có tác động mạnh đến hiệu quả kỹ thuật hay hiệu quả hoạt động NHTMCP, cụ thể:

Quy mô ngân hàng (tổng tài sản - LnAssets): Nhân tố này không có ý nghĩa thống kê ở cả hai phương pháp với mức ý nghĩa $1 \%$. Tuy nhiên, ở cả hai kết quả quy mô ngân hàng có xu hướng tác động cùng chiều với HQKT, nghĩa là hiệu quả hoạt động của các NHTMCP tại Việt Nam giai đoạn 2011 - 2018 tăng khi tổng tài sản của các ngân hàng tăng. Các ngân hàng cần cân nhắc kỹ trong việc tăng vốn để mở rộng quy mô hoạt động của mình vì có thể sẽ dẫn đến hiện tượng VRS, đặc biệt là những NHTMCP có tổng tài sản lớn.

Lợi nhuận sau thuế trên tổng tài sản (ROA): Kết quả hồi quy cho thấy với biến phụ thuộc là TE_DEA, ROA có mối quan hệ ngược chiều với HQKT, nhưng lại không có ý nghĩa thống kê. Còn đối với biến phụ thuộc là TE_SFA, ROA có mối quan hệ cùng chiều với HQKT và có ý nghĩa thống kê cao. Nghĩa là, nếu tỷ lệ ROA của các NHTMCP tăng thì hiệu quả hoạt động của các ngân hàng tăng. Cụ thể, với các yếu tố khác không thay đổi, tỷ lệ ROA tăng $1 \%$ thì HQKT của các NHTMCP tại Việt Nam tăng 8,459\% với độ tin cậy $99 \%$.

Tỷ lệ nợ xấu (NPL): Kết quả hồi quy cho thấy với biến phụ thuộc là TE_DEA, nếu tỷ trọng nợ xấu trên tổng dư nợ của NHTMCP tăng $1 \%$ thì HQKT tăng $0,430 \%$, độ tin cậy $95 \%$ trong điều kiện các yếu tố khác không thay đổi, ngược với kỳ vọng dấu. Tuy nhiên, mức độ tác động này là rất thấp $(0,430 \%)$. Bên cạnh đó, với biến phụ thuộc là TE_SFA, tỷ lệ NPL không có ý nghĩa thống kê. Do đó, chưa đủ bằng chứng khoa học mạnh mẽ chứng minh tác động giữa NPL và hiệu quả hoạt động.

Tỷ lệ tiền gửi khách hàng trên cho vay $(D L R)$ : Kết quả nghiên cứu chỉ ra rằng DLR không có ý nghĩa thống kê với cả hai biến phụ thuộc TE_DEA và TE_SFA ở mức ý nghĩa 1\%.

Tỷ lệ vốn chủ sở hũu trên tổng tài sản (ETA): Nhân tố này có ý nghĩa thống kê ở cả hai mô hình và có xu hướng tác động tích cực, phù hợp với kỳ vọng dấu. Với biến phụ thuộc là TE_DEA, với độ tin cậy là 90\%, trong điều kiện khác không thay đổi, khi tỷ lệ ETA tăng 1\% thì HQKT hay hiệu quả hoạt động của NHTMCP tăng $0,136 \%$. Đối với biến phụ thuộc là TE_SFA, trong điều kiện khác không thay đổi và độ tin cậy cao là $95 \%$, ETA tăng $1 \%$ thì hiệu quả hoạt động ngân hàng tăng $0,810 \%$.

\section{Kết luận}

Bằng việc sử dụng dữ liệu thu thập của mẫu 19 NHTMCP tại Việt Nam giai đoạn 2011 2018, nghiên cứu đã đưa ra được mức hiệu quả kỹ thuật của 19 ngân hàng này thông qua hai phương pháp chính là phương pháp phân tích bao dữ liệu (DEA) và phương pháp phân tích biên ngẫu nhiên (SFA). Với phương pháp SFA, giá trị hiệu quả kỹ thuật của các ngân hàng đạt trung bình giai đoạn 2011 - 2018 là 0,677 , ngân hàng đạt mức hiệu quả kỹ thuật cao nhất là VPB $(0,790)$, ngân hàng có mức hiệu quả kỹ thuật thấp nhất là STB $(0,513)$; còn đối với phương pháp DEA giá trị hiệu quả trung bình là 0,961 ; trong đó ngân hàng đạt hiệu quả kỹ thuật cao nhất và thấp nhất lần lượt là $\mathrm{ACB}(1,000), \operatorname{MBB}(1,000), \mathrm{TPB}(1,000), \operatorname{VCB}(1,000)$ và $\operatorname{LPB}(0,901)$. Nhóm các ngân hàng đạt mức hiệu quả kỹ thuật cao là những ngân hàng luôn tiên phong trong việc áp dụng thay đổi tiến bộ công nghệ, có mức nhận diện thương hiệu trên thị trường cao trong những năm gần đây, đặc biệt là những ngân hàng đã công bố áp dụng thành công chuẩn Basel II trong năm 2019 (ngân hàng ACB, TPB, VCB, MBB, VPB).

Ngoài ra, kết quả nghiên cứu cũng cho thấy mô hình Pooled OLS phù hợp với biến phụ thuộc là TE_DEA (hiệu quả kỹ thuật đo lường bằng phương pháp DEA), mô hình REM thích hợp 
sử dụng với biến phụ thuộc là TE_SFA (hiệu quả kỹ thuật thuật đo lường bằng phương pháp SFA). Các biến phù hợp có thể đại diện cho tổng thể, độ tin cậy cao bao gồm biến ROA, ETA và có tác động cùng chiều đến hiệu quả hoạt động NHTMCP trong giai đoạn 2011 - 2018. Trong đó, ROA có mức độ tác động lớn đến hiệu quả ngân hàng với độ tin cậy cao (khi tăng lợi nhuận trên tổng tài sản thêm $1 \%$ thì hiệu quả kỹ thuật tăng 8,459\% với độ tin cậy $99 \%$ trong điều kiện các yếu tố khác không thay đổi). Bên cạnh đó, chỉ số ETA cũng cho thấy chỉ cần tăng $1 \%$ tỷ lệ vốn chủ sở hữu trên tổng tài sản, hiệu quả kỹ thuật ngân hàng tăng $0,810 \%$ với độ tin cậy $95 \%$ trong điều kiện các yếu tố khác không thay đổi. Do đó, có thể kết luận rằng yếu tố quản lý (thể hiện qua chỉ số ROA và ETA) có tác động mạnh mẽ đến hiệu quả trong hoạt động của các NHTMCP tại Việt Nam, những ngân hàng nào có chính sách quản lý tốt, sử dụng tài sản kiếm lời hiệu quả, khả năng thanh khoản cao, tỷ lệ an toàn vốn càng cao và áp dụng tiến bộ khoa học công nghệ tiên tiến vào quy trình hoạt động thì hiệu quả hoạt động càng tốt.

\section{Tài liệu tham khảo}

Aigner, D. L., \& Chu, S. F. (1968). On estimating the industry production function. The American Economic Review, 4, 826-839.

Aigner, D. L., Lovell, C. K., \& Schmidt, P. (1977). Formulation and estimation of stochastic frontier production models. Journal of Econometrics, 6(1), 21-37.

Berger, A. N., \& Humphrey, D. B. (1997). Efficiency of financial institutions: International survey and directions for future research. European Journal of Operational Research, 98(2), 175212.

Charnes, A., Cooper, W. W., \& Banker, R. D. (1984). Some models for estimating technical and scale inefficiencies in data envelopment analysis. Management Science, 30(9), 1078-1092.

Charnes, A., Cooper, W. W., \& Rhodes, E. (1978). Mearsuring the efficiency of decision making units. European Journal of Operation Research, 2, 429-444.

Chen, C. P., Hu, J. L., \& Su, Y. Y. (2006). Owership reform and efficiency of nationwide banks in China. Retrieved Octoter 20, 2019, from http://faculty.washington.edu/karyiu/confer/ beijing06/papers/hu-chen-su.pdf

Coelli, D. S. P., O’ Donnell, C. J., \& Battese, G. E. (2005). An introduction to efficiency and productivity analysis. Berlin, Germany: Springer Science \& Business Media.

Eva, H. (2018). Technical efficiency of bank in Central and Eastern Europe. International Journal of Financial Studies, 6(3), 66.

Farrell, M. J. (1957). The measurement of productive efficiency. Journal of Royal Statistical Society, 120(3), 253-290.

Faello, J. (2015). Understanding the limitations of financial ratios. Academy of Accounting and Financial Studies Journal, 19(3), 75-86.

Gamachis, G. (2016). Technical efficiency and productivity of Ethiopian Commercial Banks: Data encelopment analysis (DEA) approach. International Journal of Scientific and Research Publications, 6, 860-864.

Huynh, T. T. P. (2015). Ước lượng hiệu quả hoạt động kinh doanh của các Ngân hàng thương mại Việt Nam [Estimating the business performance of Vietnamese commercial banks]. Tạp chí Khoa học Công nghệ \& Thục Phẩm, 7, 51-57. 
Inès, A. (2013). Determinants of Tunisian bank efficiency: A DEA analysis. International Journal of Financial Research, 4(4), 128-139.

Kodde, D., \& Palm, F. (1986). Wald criteria for jointly testing equality and inequality restrictions. Technology and Investment, 5(2).

Koopmans, C. T. (1957). Three essays on the state of economic science. New York, NY: McGrawHill Book Company, Inc.

Michael, A. (2016). Determinants of bank technical efficiency: Evidence from rural and community banks in Ghana. Cogent Business \& Management, 3.

Ngân hàng Nhà nước Việt Nam. (2018). Danh sách các Ngân hàng thuoong mại cổ phần tại Việt Nam [List of joint stock commercial banks in Vietnam]. Retrieved Octorber 21, 2019, from https://www.sbv.gov.vn

Nguyen, K. M. (2015). Tài chính doanh nghiệp căn bản [Basic corporate finance]. Hanoi, Vietnam: NXB Tài chính.

Nguyen, H. M., \& Nguyen, T. C. (2012). Hiệu quả hoạt động của ngân hàng tại các nước Đông Nam Á và bài học kinh nghiệm cho Việt Nam [Performance of banks in Southeast Asian countries and lessons for Vietnam]. Tạp chí Kinh tế và Chính trị thế giới, 11(199).

Nguyen, K. M., \& Nguyen, T. T. T. (2019). Ảnh hưởng của mở rộng chi nhánh đến hiệu quả hoạt động của các Ngân hàng thương mại: Bằng chứng nghiên cứu thực nghiệm tại Việt Nam [Effect of branch expansion on commercial banks' performance: empirical evidence in Vietnam]. Tạp chí Kinh tế và Ngân hàng châu Á, 159.

Nguyen, P. N. (2013). Giáo trình phân tích báo cáo tài chính [Textbook analysis of financial statements]. Hanoi, Vietnam: NXB Đại học Kinh tế Quốc dân.

Nguyen, H. V. (2008). Phân tích các nhân tố ảnh hưởng đến hiệu quả hoạt động của các Ngân hàng thuoong mại Việt Nam [Analysis of factors affecting the performance of commercial banks in Vietnam] (Doctoral dissertation, National Economics University, Hanoi, Vietnam). Retrieved October 22, 2019, from https://khotrithucso.com/doc/p/phan-tich-cac-nhan-toanh-huong-den-hieu-qua-hoat-dong-cua-219234

Nguyen, N. T. (2018). Phân tích các nhân tố ảnh hưởng đến hiệu quả kinh doanh của các Ngân hàng thuơng mại cổ phần Việt Nam [Analysis of factors affecting the business performance of joint stock commercial banks in Vietnam]. Retrieved October 23, 2019, from https://www.slideshare.net/trongthuy3/de-tai-nhan-to-anh-huong-den-hieu-qua-kinhdoanh-cua-ngan-hang-thuong-mai

Nguyen, T. T. T. (2017). Hiệu quả hoạt động của các Ngân hàng thương mại trên địa bàn tỉnh Thái Nguyên [Operational efficiency of commercial banks in Thai Nguyen province]. Tạp chí Khoa học Trưòng Đại học Cần Tho, 50(D), 52-62.

Nguyen, K. Q. (2016). Xây dựng mô hình DEA đánh giá hoạt động của các Ngân hàng thương mại Việt Nam [Building a DEA model to evaluate the performance of Vietnamese commercial banks]. Tạp chí Tài chính, 2 .

Nguyen, T. T. H., \& Le, V. H. (2018). Phân tích hiệu quả hoạt động của hệ thống ngân hàng thương mại cổ phần Việt Nam giai đoạn 2011-2016 [Analyzing the performance of the joint stock commercial banking system in Vietnam in the period 2011-2016]. Tạp chí Kinh tế đối ngoại, 103. 
Pham, T. L., \& Chau, H. T. K. (2011). Hiệu quả kỹ thuật của các Ngân hàng thương mại Việt Nam [Technical efficiency of Vietnamese commercial banks]. Tạp chí Công nghệngân hàng, 69, 20-26.

Phan, N. T. H., \& Tran, T. P. (2017). Hiệu quả kinh doanh của các Ngân hàng thương mại Việt Nam sau sáp nhập, hợp nhất, mua lại: Tiếp cận phương pháp DEA [Business performance of Vietnamese commercial banks after mergers, consolidations and acquisitions: DEA approach]. Tạp chí Ngân hàng, 24.

Rose, P. S., \& Hudgins, S. C. (2008). Bank management and financial services. New York, NY: The McGraw-Hill Companies, Inc. 